\title{
[gw22-e0576] NOVEL MINIATURE MOBILE CARDIAC CATHETERISATION LABORATORY FOR ST-ELEVATION MYOCARDIAL INFARCTION FOLLOWING NATURAL DISASTER: A FEASIBILITY STUDY
}

Liang Zhuo, ${ }^{1}$ Yao Tian-ming, ${ }^{2}$ Sun Jing-yang, ${ }^{2}$ Wang Geng, ${ }^{2}$ Huo $\mathrm{Yu}^{2}$ Han Ya-ling ${ }^{1}$ Department Of Cardiology, Shenyang Northern Hospital, Shenyang, China; ${ }^{2}$ Department Of Cardiology, Shenyang Northern Hospital, Shenyang, China

10.1136/heartjnl-2011-300867.428

Purpose A novel miniature mobile cardiac catheterisation laboratory (Mini Mobile Cath Lab) was developed to provide emergency interventional services for ST-Elevation Myocardial Infarction (STEMI) patients in natural disaster medical rescue. Animal experiment was completed in the Mini Mobile Cath Lab in hypothetical natural disaster striken area for feasibility study on the use of the Mini Mobile Cath Lab.

Methods The Mini Mobile Cath Lab was transported to the hypothetical natural disaster striken area by truck for medical rescue. Coronary angiography and primary PCI were provided for dogs. Parameters of the Mini Mobile Cath Lab in transportation and transformation were monitored. Functions of the Mini Mobile Cath Lab were evaluated after transportation through animal experiment.

Results The Mini Mobile Cath Lab was transported to the hypothetical natural disaster striken area by truck in $100 \mathrm{~min}$ at the average speed of $85 \mathrm{~km} / \mathrm{h}$ on mountain road in the winter day under a light snow $\left(-15 \sim-20^{\circ} \mathrm{C} /-68 \sim-59^{\circ} \mathrm{F}\right)$. After the transformation from carrying form to unfolding form in 5 min successfully, the Mini Mobile Cath Lab was quickly prepared to be qualified for receiving patients within $20 \mathrm{~min}$ in the wetland area. Coronary angiography for dogs was successfully conducted in animal experiment. Fluoroscopy images of all necessary angles were preserved. The widest angle of the medium C-arm for fluoroscopy reached LAO60\%/RAO60\% CRA $45^{\circ} / \mathrm{CAU} 45^{\circ}$, which is necessary for angiography and PCI. Dogs with STEMI underwent percutaneous intracoronary stent implantation, and re-canalisation was achieved with a TIMI flow grade three after stent implantation. IABP was successfully inserted in the dog that suffered from cardiogenic shock. Spontaneous circulation was achieved after resuscitation in the two dogs that suffered from ventricular fibrillation (VF). The total door-to-balloon time was $68 \pm 5.0$ min, which even included the time spent in producing animal model, IABP insertion and resuscitation procedures. Function of all equipments in the Mini Mobile Cath Lab was proved to be stable after long distance transportation on mountain road through the animal experiment. The weather condition and ground type in the experiment did not influence functions of the Mini Mobile Cath Lab.

Conclusions The feasibility of emergency interventional treatment for patients with STEMI provided by the Mini Mobile Cath Lab in natural disaster medical rescue was preliminarily proved. The Mini Mobile Cath Lab may play an important role in reducing the transfer-related time and door-to-balloon time for primary PCI for patients with STEMI resulted from psychological stress, undue fatigue, trauma or atherosclerotic 
factors in disaster medical rescue, because it can have medical capacity close to natural disaster striken area when tradition services are not available. 